\title{
CARACTERÍSTICAS CLÍNICOEPIDEMIOLÓGICAS DE PACIENTES FALLECIDOS POR COVID-19 EN UN HOSPITAL NACIONAL DE LIMA, PERÚ
}

\author{
CLINICOEPIDEMIOLOGICAL CHARACTERISTICS OF PATIENTS WHO DIED FROM COVID-19 AT A \\ NATIONAL HOSPITAL OF LIMA, PERU

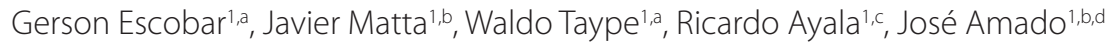

\begin{abstract}
RESUMEN
Introducción: La pandemia debida a enfermedad por coronavirus 2019 (COVID-19) ha producido más de 70 mil muertes en el mundo. Objetivo: Describir las características de pacientes fallecidos por COVID-19 en un hospital terciario. Métodos: Estudio descriptivo realizado en el servicio de emergencia del hospital Rebagliati Lima-Perú, que incluye los pacientes fallecidos con resultado positivo a infección por SARS-CoV-2 mediante RT-PCR hasta el 4 de abril de 2020. Se revisó la historia clínica y registros hospitalarios buscando variables sociodemográficas, antecedentes, manifestaciones clínicas, radiológicas, tratamiento y evolución. Resultados: Se identificaron 14 casos, 78,6\% de sexo masculino, edad promedio 73,4 años (rango 26 a 97). Adquirieron la infección en el exterior del país el $21,4 \%$ de casos. Se encontró factores de riesgo en $92,9 \%$ de pacientes (más frecuentes adulto mayor, hipertensión arterial y obesidad). Los síntomas más frecuentes fueron disnea, fiebre y tos, con tiempo de enfermedad 8 días (+/-3,0); los signos polipnea y estertores respiratorios. Los hallazgos de laboratorio más frecuentes fueron proteína C reactiva elevada (promedio $22 \mathrm{mg} / \mathrm{dL}$ ) e hipoxemia. La presentación radiológica predominante fue infiltrado pulmonar intersticial bilateral en vidrio esmerilado. Ingresaron a ventilación mecánica 78,6\% (11 de 14 casos); recibió azitromicina $71,4 \%$, hidroxicloroquina 64,3\% y antibióticos de amplio espectro $57,1 \%$ de los casos; con estancia hospitalaria de 4,7 días (+/-2,4). Conclusión: Los fallecidos por COVID-19 presentaron neumonía grave bilateral, más frecuentes en varones, con factores de riesgo (adulto mayor, hipertensión arterial y obesidad), con alta necesidad de asistencia ventilatoria.
\end{abstract}

Palabras clave: COVID-19; Neumonía viral; SARS-CoV-2 (fuente: DeCS BIREME).

\begin{abstract}
Introduction: The COVID-19 pandemic has caused more than 70 thousand deaths worldwide. Objective: To describe the characteristics of COVID-19 patients who died in a tertiary hospital. Methods: A descriptive study was carried out in the emergency service of the Hospital Rebagliati in Lima, Peru, which includes deceased patients with a positive result for SARS-CoV-2 infection diagnosed by RT-PCR until April 4, 2020. The medical history was reviewed. and hospital records looking for sociodemographic variables, clinical characteristics, radiological manifestations, treatment and evolution. Results: 14 cases were identified, $78.6 \%$ were male, average age 73.4 years (range 26 to 97). 21.4\% of cases acquired the infection out of Peru. Risk factors were found in $92.9 \%$ of patients (more frequent elderly, hypertension and obesity). The most frequent symptoms were dyspnea, fever and cough, with illness time 8 days (+/- 3); signs of polypnea and respiratory rales. The most frequent laboratory findings were elevated C-reactive protein (average $22 \mathrm{mg} / \mathrm{dL}$ ) and hypoxemia. The predominant radiological presentation was bilateral interstitial pulmonary infiltration in ground glass. $78.6 \%$ (11 of 14 cases) entered mechanical ventilation; $71.4 \%$ of the cases received azithromycin, $64.3 \%$ hydroxychloroquine and $57.1 \%$ broad-spectrum antibiotics; with a 4.7 day hospital stay (+/-2.4). Conclusion: Those who died from COVID-19 presented bilateral severe pneumonia, more frequent in men, with risk factors (elderly, hypertension and obesity), with a high need for ventilatory assistance.
\end{abstract}

Key words: COVID-19; Viral pneumonia; SARS-CoV-2 (source: MeSH NLM).

\footnotetext{
${ }^{1}$ Hospital Edgardo Rebagliati Martins, Lima-Perú.

${ }^{2}$ Universidad Nacional Mayor de San Marcos, Lima-Perú.

a Médico especialista en emergencias y desastres, ${ }^{b}$ Médico internista, ${ }^{c}$ Médico residente de emergencias y desastres, ${ }^{d}$ Doctor en medicina.
}

Citar como: Gerson Escobar, Javier Matta, Waldo Taype, Ricardo Ayala, José Amado. Características clínicoepidemiológicas de pacientes fallecidos por covid-19 en un hospital nacional de Lima, Perú. Rev. Fac. Med. Hum. Abril 2020; 20(2):180-185. DOI 10.25176/RFMH.v20i2.2940 


\section{INTRODUCCIÓN}

La nueva enfermedad asociada a coronavirus originada en China el 2019, designada COVID-19 (Coronavirus Disease 2019) es producida por un nuevo virus denominado Severe acute respiratory syndrome coronavirus 2 (SARS-CoV-2), de presunto origen zoonótico en Asia, pero con evidente propagación de persona a persona que se ha diseminado rápidamente en el mundo y declarada oficialmente como pandemia por la Organización Mundial de la Salud el 11 de marzo de $2020^{(1-4)}$.

Esta enfermedad se caracteriza por producir neumonía severa progresiva, notificándose más de 80 mil casos confirmados y más de 3000 fallecidos en China hasta febrero de este año. En el mundo se reportan alrededor de un millón de infectados de los cuales han fallecido más de 67 mil personas, siendo los países más afectados Italia, España y Francia ${ }^{(1,4-6)}$.

El primer caso reportado en América fue en Estados Unidos de Norteamérica (EEUU) el 23 de enero de 2020, pero recién a fines de marzo el número de casos se incrementa abruptamente, considerandos actualmente el nuevo epicentro de la pandemia. En América del Sur se presentó primero en Argentina en febrero de 2020, siendo actualmente los países más afectados de esta parte del continente Brasil, Chile y Ecuador ${ }^{(5,6)}$.

En nuestro país, se confirmóla primera persona infectada el 6 de marzo de 2020, incrementándose rápidamente el número de casos, se presentan las primeras víctimas mortales el 19 de marzo (tres el mismo día) y dos en los días siguientes. En Perú hasta el 6 de abril de 2020 se realizaron 20414 pruebas diagnósticas, obteniendo 2561 casos positivos para SARS-CoV-2, 387 pacientes estaban hospitalizados, 89 en cuidados intensivos con ventilación mecánica y se habían reportado 92 fallecimientos ${ }^{(5,6)}$.

El Hospital Nacional Edgardo Rebagliati perteneciente al Seguro Social de Salud (EsSalud) fue considerado como centro de referencia para COVID-19 en Lima y viene atendiendo una importante cantidad de pacientes hospitalizados en el país, habiendo sobrepasado la capacidad de los ambientes destinados para este fin. El presente estudio busca describir las características demográficas, clínicas y laboratoriales de pacientes con COVID-19 fallecidos en el servicio de emergencia de un hospital terciario de la seguridad social.

\section{MÉTODOS}

\section{Diseño y área de estudio}

Estudio observacional, retrospectivo realizado en el servicio de emergencia de adultos del Hospital Nacional Edgardo Rebagliati Martins de EsSalud, ubicado en el distrito de Jesús María en Lima-Perú, incluyendo los pacientes fallecidos con resultado positivo a infección por SARS-CoV-2 (mediante RT-PCR) entre el 6 de marzo y 4 de abril del 2020.

\section{Población y muestra}

Se incluyó al total de pacientes fallecidos con resultado positivo a infección por SARS-CoV-2 (mediante RT-PCR) entre el 6 de marzo y 4 de abril del 2020. En el hospital se destinó un área aislada para identificación, atención y hospitalización de pacientes COVID-19 desde los primeros días de marzo del 2020. Según las características el paciente era enviado a casa para aislamiento, pasaba a un tópico de emergencia o era admitido a salas de observación, dispuestas para pacientes sospechosos, confirmados y críticos (con ventilación mecánica).

\section{Procedimientos y variables}

Se identificaron a los pacientes con diagnóstico de COVID-19 que hayan fallecido dentro del Hospital, una vez identificados, se revisaron las historias clínicas y registros hospitalarios buscando variables sociodemográficas, antecedentes, manifestaciones clínicas, radiológicas, tratamiento indicado y evolución.

\section{Análisis estadístico}

Los datos fueron codificados y procesados en Microsoft Excel 2013, se realizó un análisis estadístico descriptivo, calculando medidas de tendencia central y de dispersión para las variables cuantitativas; así como la distribución de frecuencias absolutas y relativas, en el caso de las variables cualitativas.

\section{Consideraciones éticas}

Se cumplieron con los principios de Helsinki, los datos que permitían la identificación de los pacientes fueron encriptados para proteger su confidencialidad por solo un miembro del equipo de investigación. Se obtuvo autorización de la jefatura del servicio.

\section{RESULTADOS}

Se identificaron 14 pacientes con COVID-19 fallecidos entre 11 y 31 de marzo. La edad de los pacientes varió entre 26 y 97 años (promedio 73,4 +/- 40,7), siendo del sexo masculino el $78,6 \%$ de los casos, la distribución por edad y sus características epidemiológicas se presentan en la Tabla 1. Uno de los pacientes no fue hospitalizado y falleció en su domicilio dos días después de haberse atendido en el servicio de emergencia, conociéndose su resultado positivo de hisopado nasofaríngeo el mismo día del fallecimiento.

Se encontraron factores de riesgo para enfermedad grave en $92,9 \%$ de los casos, siendo más frecuentes la edad mayor de 60 años, hipertensión arterial y obesidad. Los distritos de procedencia más frecuentes fueron 
Miraflores, Jesús María, Chorrillos y La Molina. Dos pacientes adquirieron la infección en España y uno en Estados Unidos de Norteamérica.

Se reportó un tiempo de enfermedad entre 3 y 14 días (promedio 8 +/- 3,0). Las manifestaciones clínicas más frecuentes al ingreso fueron polipnea, disnea, fiebre, tos y estertores pulmonares (Tabla 2); los diagnósticos de ingreso fueron insuficiencia respiratoria aguda y neumonía severa en todos los casos. Las alteraciones de exámenes de laboratorio más frecuentes fueron elevación de proteína $C$ reactiva, hipoxemia y linfopenia (Tabla 2).

Se realizó radiografía de tórax en 9 pacientes y tomografía pulmonar en 2 casos, no habiéndose realizado estudios en los primeros casos por no disponer de equipos o disposiciones específicas para trasladar a los pacientes al área de radiodiagnóstico. Se encontraron alteraciones radiológicas bilaterales en todos los casos, predominando patrón intersticial en vidrio esmerilado (en 6 de los 9 casos) y patrón mixto alveolar e intersticial en los otros 3 casos (Figura 1).

En lo referente al tratamiento, 11 casos $(78,6 \%)$ ingresaron a ventilación mecánica, uno de ellos en modalidad no invasiva en un paciente de 97 años con adecuado nivel de conciencia, pero sin respuesta favorable. Se administró azitromicina 500 mg VO diarios en 10 pacientes $(71,4)$, hidroxicloroquina a dosis de 400 mg VO cada 12 horas en 9 casos (64,3\%) y antibióticos de amplio espectro en $57,1 \%$. Todos los pacientes recibieron oxígeno suplementario y sintomáticos como analgésicos (paracetamol) o antipiréticos (metamizol) condicionales. Se administró sedación (midazolam) y analgesia (fentanilo) por infusión endovenosa continua a 9 de los 11 pacientes en ventilación mecánica. Se usó corticoides sistémicos (hidrocortisona) en un paciente con antecedente de asma y evolución desfavorable.

Las complicaciones reportadas fueron shock distributivo en apoyo inotrópico con noradrenalina (cuatro casos), disfunción orgánica múltiple (dos casos), falla renal aguda con hemodiálisis de apoyo (un caso), paro cardiorespiratorio al ingreso que responde a resucitación (un caso) y neumotórax posterior a procedimiento resuelto con drenaje torácico (un caso). La estancia hospitalaria promedio fue 4,7 días (+/-2,4).

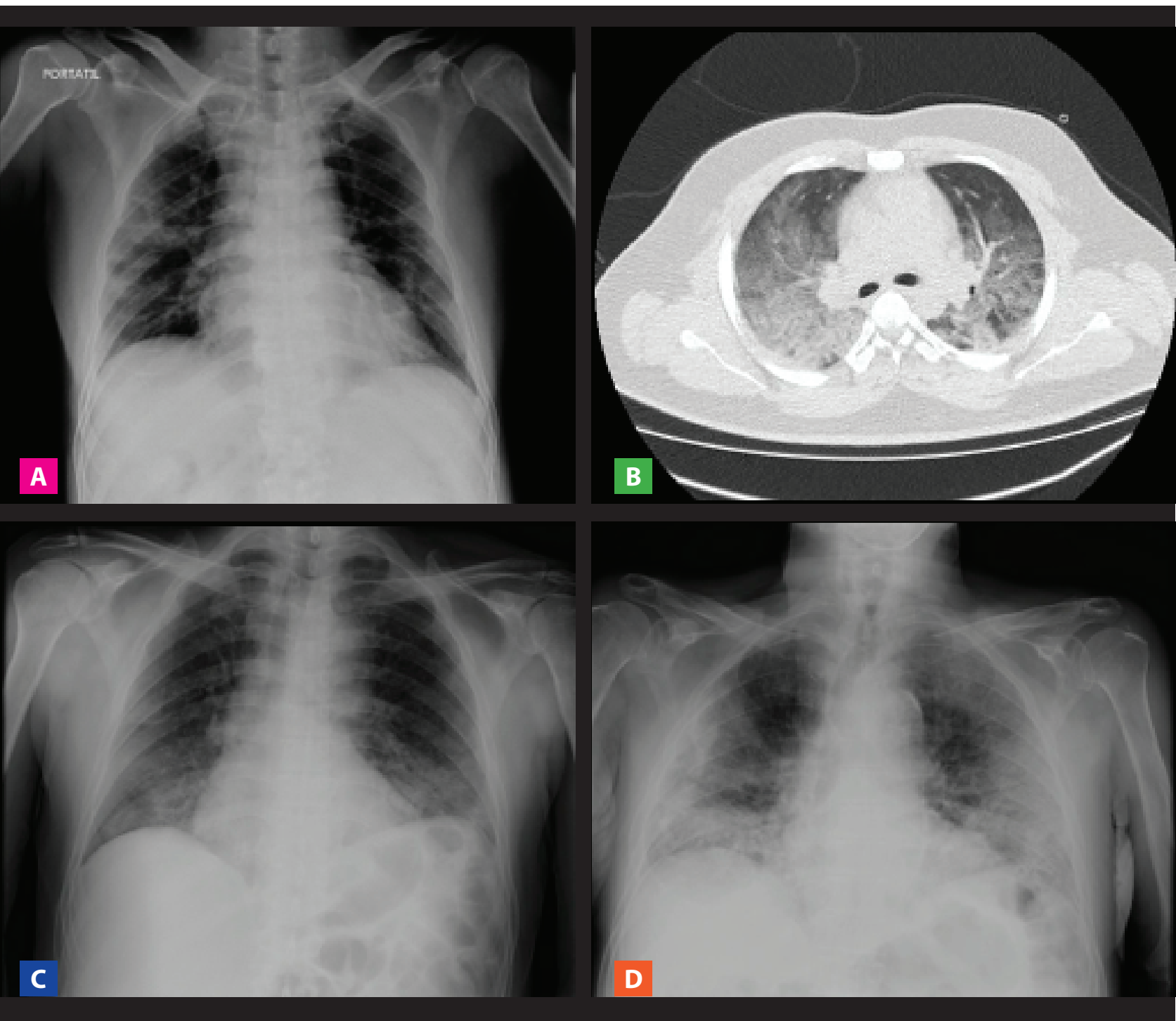

Figura 1. Estudios radiológicos de pacientes fallecidos por COVID-19, en un hospital terciario de Lima, Perú. 
(A) Varón 61 años con diabetes e hipertensión arterial, vidrio esmerilado. (C) Varón 66 años sin antecedentes muestra patrón intersticial en vidrio esmerilado bilateral. y con patrón alveolo-intersticial bilateral. (D) Mujer 97 (B) Varón 26 años antecedente de asma bronquial y años antecedente de hipertensión arterial, con lesiones obesidad, con predominio de lesiones intersticiales en alveolo-intersticiales bilaterales.

Tabla 1. Características epidemiológicas de pacientes fallecidos por COVID-19, en un hospital terciario de Lima hasta el 6 de abril de 2020.

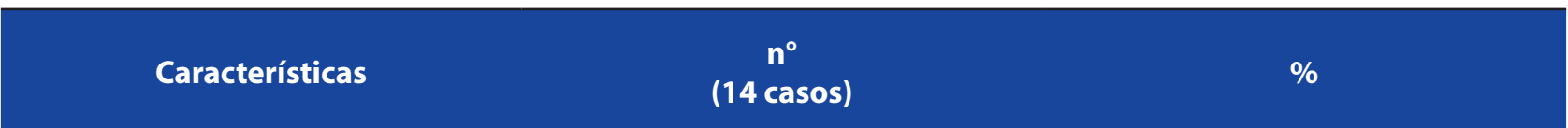

\section{Edad}

Menor de 30 años

30 a 39 años

40 a 49 años

50 a 59 años

60 años o más

\section{Sexo}

Masculino

Femenino

\section{Distrito de procedencia}

Lima Centro

Otra zona de Lima

\section{Lugar de infección}

Extranjero

Local

\section{Factores de riesgo}

Adulto mayor

Hipertensión arterial

Obesidad

Enfermedad pulmonar crónica

Diabetes mellitus

Uso de corticoides

Infección por VIH

\section{$\mathbf{N}^{\circ}$ de factores de riesgo por paciente}

Uno

Dos 
Tabla 2. Manifestaciones clínicas y resultados de exámenes auxiliares de pacientes fallecidos por COVID-19, en un hospital terciario de Lima hasta el 6 de abril de 2020.

Manifestaciones clínicas y resultados de laboratorio al ingreso

$\mathrm{n}^{\circ}$

(14 casos)

\section{Síntomas}

Fiebre

$11 \quad 78,6$

Disnea

$11 \quad 78,6$

Tos

$10 \quad 71,4$

Diarrea

428,6

Rinorrea y odinofagia

$2 \quad 14,3$

Náuseas y vómitos
$\%$

14,3

\section{Signos}

Polipnea (> 20 respiraciones por minuto)

Estertores respiratorios

12

64,3

Saturación de oxígeno $<90 \%$

\section{Exámenes auxiliares}

$\mathrm{PCR}^{\mathrm{a}}$ elevada (media $22 \mathrm{mg} / \mathrm{dl}+/-4,8$ )

$\mathrm{PaFi}<240$

Linfopenia $(<1000 / \mathrm{mm} 3)$

Leucocitosis (>12000/mm3)

Elevación de transaminasas (>40 U/L)

Hiperglicemia (>140 mg/dl)

a: PCR: Proterina $C$ reactiva ultrasensible.

\section{DISCUSIÓN}

Las características de los pacientes fallecidos por COVID-19 descritos corresponden a los primeros ocurridos en el hospital de estudio durante el desarrollo de la pandemia en el Perú. Similar a los casos graves reportados en China y EEUU, indican una forma de enfermedad predominante en varones que después de más de una semana de fiebre, disnea o tos desarrollan insuficiencia respiratoria aguda por neumonía grave y fallecen 4 días después a pesar de recibir ventilación mecánica(7-10).

La edad mayor a 60 años, comorbilidades como hipertensión arterial y obesidad fueron factores de riesgo reportados para desarrollar la forma grave de enfermedad similares a lo reportado en China y EEUU. Un solo paciente no presentó ningún factor de riesgo, pero un porcentaje importante presentó más de uno asociándose a mayor mortalidad. Se encontró un paciente de 26 años que evoluciona desfavorablemente y fallece, pero tenía antecedentes de obesidad y asma bronquial, factores de mal pronóstico para esta enfermedad ${ }^{(7,9,10)}$.

La fiebre y disnea son síntomas frecuentes, pero no suficientes como criterio diagnóstico o de severidad, siendo necesarios asociarlos a datos objetivos como saturación de oxígeno capilar o exámenes auxiliares para tomar acciones en el paciente. Tener presente que síntomas gastrointestinales como diarrea y vómitos son consideradas formas de presentación atípicas (poco frecuente), sin embargo fueron reportadas en el $28 \%$ de esta serie de $\operatorname{casos}^{(7,10,11)}$.

Asociado a los factores de riesgo se deben utilizar datos objetivos importantes para la valoración del paciente, destacando el uso de saturación de oxígeno, frecuencia respiratoria, presión arterial sistólica y estado de conciencia (valorado por la escala de Glasgow), estos tres últimos forman parte del qSOFA (quick Sequential Organ Failure Assessment) ${ }^{(12)}$. Indicadores que se pueden utilizar en los pacientes que consultan emergencia y así disminuir la probabilidad de tener pacientes fallecidos en el domicilio sin vigilancia ni tratamiento oportuno.

En los primeros casos hubo problemas para realizar exámenes auxiliares en el área de COVID-19, para evitar estos contratiempos es necesario planificar e implementar oportunamente estas áreas. En los hallazgos se encontró incremento de reactantes de fase aguda (proteína $C$ reactiva) y linfopenia, similar a lo reportado en Asia y Norteamérica(7,10,13,14). 
Se evidencia lesiones pulmonares multifocales y bilaterales, similar a lo reportado en China, Corea, Francia y EEUU predominando la lesión intersticial característica de neumonía viral(9,10,15-17). Sin embargo debemos remarcar la falta de estudios adecuados en la mayoría de pacientes, pues una semana después de recibir los primeros casos recién se contaba con un equipo portátil de radiografías y era muy difícil realizar tomografías a los pacientes una vez internados en el área de aislamiento por falta de continuidad física con los servicios de diagnóstico por imágenes.

La combinación hidroxicloroquina y azitromicina fue el tratamiento más frecuente, a pesar de que aun las evidencias disponibles no son suficientes ${ }^{(18)}$. Inicialmente no estaba autorizado el uso de hidroxicloroquina, pero días después fue autorizado en el hospital. El soporte ventilatorio invasivo al inicio también tuvo problemas por disponibilidad de equipos y ambientes adecuados, pero después se consiguió lo necesario, a pesar de lo cual no se obtuvo buena respuesta clínica por el gran compromiso pulmonar que produce la infección, similar a lo reportado en otros países.

Entre las limitaciones del estudio está el pequeño número de casos, datos clínicos y resultados de exámenes auxiliares incompletos en algunos pacientes y falta de estudios para infección concomitante con otros virus o complicaciones bacterianas. Sin embargo es un reporte inicial que muestra las consecuencias de la pandemia de COVID-19 en un hospital peruano.

\section{CONCLUSIÓN}

En conclusión, este reporte presenta las características de los primeros pacientes fallecidos por COVID-19 en Perú quienes desarrollaron insuficiencia respiratoria aguda por neumonía grave, más frecuente en el sexo masculino, con edad avanzada, hipertensión arterial y obesidad como factores de riesgo y alta necesidad de asistencia ventilatoria.

Contribuciones de autoría: Todos los autores han participado en la concepción y diseño del artículo; aporte de pacientes; recolección de resultados; análisise interpretación de datos; redacción del artículo; revisión crítica del artículo; aprobación de la versión final.

Financiamiento: Autofinanciado.

Conflicto de interés: Los autores declaran no tener conflicto de interés en la publicación de este artículo.

Recibido: 08 de abril 2020

Aprobado: 10 de abril 2020

Correspondencia: José Percy Amado Tineo.

Dirección: Belisario Flores 238 Dpto 301, Lince, Lima-Perú.

Teléfono: 990452547; (01)2654901.

Correo:jpamadot@gmail.com;jpamadot@gmail.com;jamadot@unmsm.edu.pe

\section{REFERENCIAS BIBLIOGRÁFICAS}

1. Ministerio de Salud. Prevención y atención de personas afectadas por COVID-19 en Perú. Lima-Perú; 2020.

2. Wu F, Zhao S, Yu B, Chen Y-M, Wang W, Song Z-G, et al. A new coronavirus associated with human respiratory disease in China. Nature. 2020;579:265-9. DOI: 10.1038/s41586-020-2008-3

3. World Health Organization. Alocución de apertura del Director General de la OMS en la rueda de prensa sobre la COVID-19 celebrada el 11 de marzo de 2020 [Internet]. Discursos del Director General de la OMS. 2020 [cited 2020 Mar 18]

4. Chen N, Zhou M, Dong X, Qu J, Gong F, Han Y, et al. Epidemiological and clinical characteristics of 99 cases of 2019 novel coronavirus pneumonia in Wuhan, China: a descriptive study. Lancet 2020;395(10223):507-13. DOI: 10.1016/S0140-6736(20)30211-7

5. World Health Organization. Coronavirus disease 2019 (COVID-19) Situation Report - 77 [Internet]. 2020 [cited 2020 Abr 6].

6. Ministerio de Salud - Perú. Minsa: Casos confirmados por coronavirus COVID-19 son 2561 en Perú Comunicado N56. 2020 [cited 2020 Apr 6]

7. Zhang J, Dong X, Cao Y, Yuan Y, Yang Y, Yan Y, et al. Clinical characteristics of 140 patients infected with SARS-CoV-2 in Wuhan, China. Allergy Eur J Allergy Clin Immunol. 2020;(February):1-12. DOI: 10.1111/all.14238

8. Guan W, Ni Z, Hu Y, Liang WH, Ou CQ, He JX, et al. Clinical Characteristics of Coronavirus Disease 2019 in China. N Engl J Med 2020 Feb 28;1-13 [Epub ahead of print]. DOI: 10.1056/NEJMoa2002032

9. Bernard S, Rolland P, Silue $Y$, Mailles A, Campese C, Simondon A, et al. First cases of coronavirus disease 2019 (COVID-19) in France: surveillance, investigations and control measures, January 2020. Euro Surveill. 2020;25(6) DOI: 10.2807/1560-7917.ES.2020.25.6.2000094

10. Bhatraju PK, Ghassemieh BJ, Nichols M, Kim R, Jerome KR, Nalla AK, et al Covid-19 in Critically III Patients in the Seattle Region - Case Series. N Engl J

\section{Med 2020 Mar 30; [Epub ahead of print]. DOI: 10.1056/NEJMoa2004500}

11. Yang X, Yu Y, Xu J, Shu H, Xia J, Liu H, et al. Clinical course and outcomes of critically ill patients with SARS-CoV-2 pneumonia in Wuhan, China: a singlecentered, retrospective, observational study. Lancet Respir Med. 2020;(20):1-7 [Epub ahead of print]. DOI: 10.1016/S2213-2600(20)30079-5

12. Ramos JGR, Da Hora R, Teixeira MB, Gobatto ALN, Coutinho RVDS, Caldas JR, et al. Prognostic ability of quick-SOFA across different age groups of patients with suspected infection outside the intensive care unit: A cohort study. J Crit Care. 2018;47:178-84. DOI: 10.1016/j.jcrc.2018.07.008

13. Shi F, Yu Q, Huang W, Tan C. 2019 Novel Coronavirus (COVID-19) Pneumonia with Hemoptysis as the Initial Symptom: CT and Clinical Features. Korean J Radiol. 2020;21:2-5

14. Xu Z, Shi L, Wang Y, Zhang J, Huang L, Zhang C, et al. Pathological findings of COVID-19 associated with acute respiratory distress syndrome. Lancet Respir Med [Internet]. 2020;(20):19-21 [Epub ahead of print]. Available from: http://dx.doi.org/10.1016/S2213-2600(20)30076-X

15. Shi $\mathrm{H}$, Han X, Zheng C. Evolution of CT Manifestations in a Patient Recovered from 2019 Novel Coronavirus (2019-nCoV) Pneumonia in Wuhan China. Radiology. 2020;295(1):20. DOI: 10.3348/kjr.2020.0181

16. Shi H, Han X, Jiang N, Cao Y, Alwalid O, Gu J, et al. Radiological findings from 81 patients with COVID-19 pneumonia in Wuhan, China: a descriptive study. Lancet Infect Dis 2020;20(4):425-34. DOI: 10.1016/S1473-3099(20)30086-4

17. Yoon SH, Lee KH, Kim JY, Lee YK, Ko H, Kim KH, et al. Chest Radiographic and CT Findings of the 2019 Novel Coronavirus Disease (COVID-19): Analysis of Nine Patients Treated in Korea. Korean J Radiol 2020;21(4):494. DOI: 10.3348/ kjr.2020.0132

18.World Health Organization. Clinical management of severe acute respiratory infection when COVID-19 is suspected. Interim guidance 13 march 2020. 